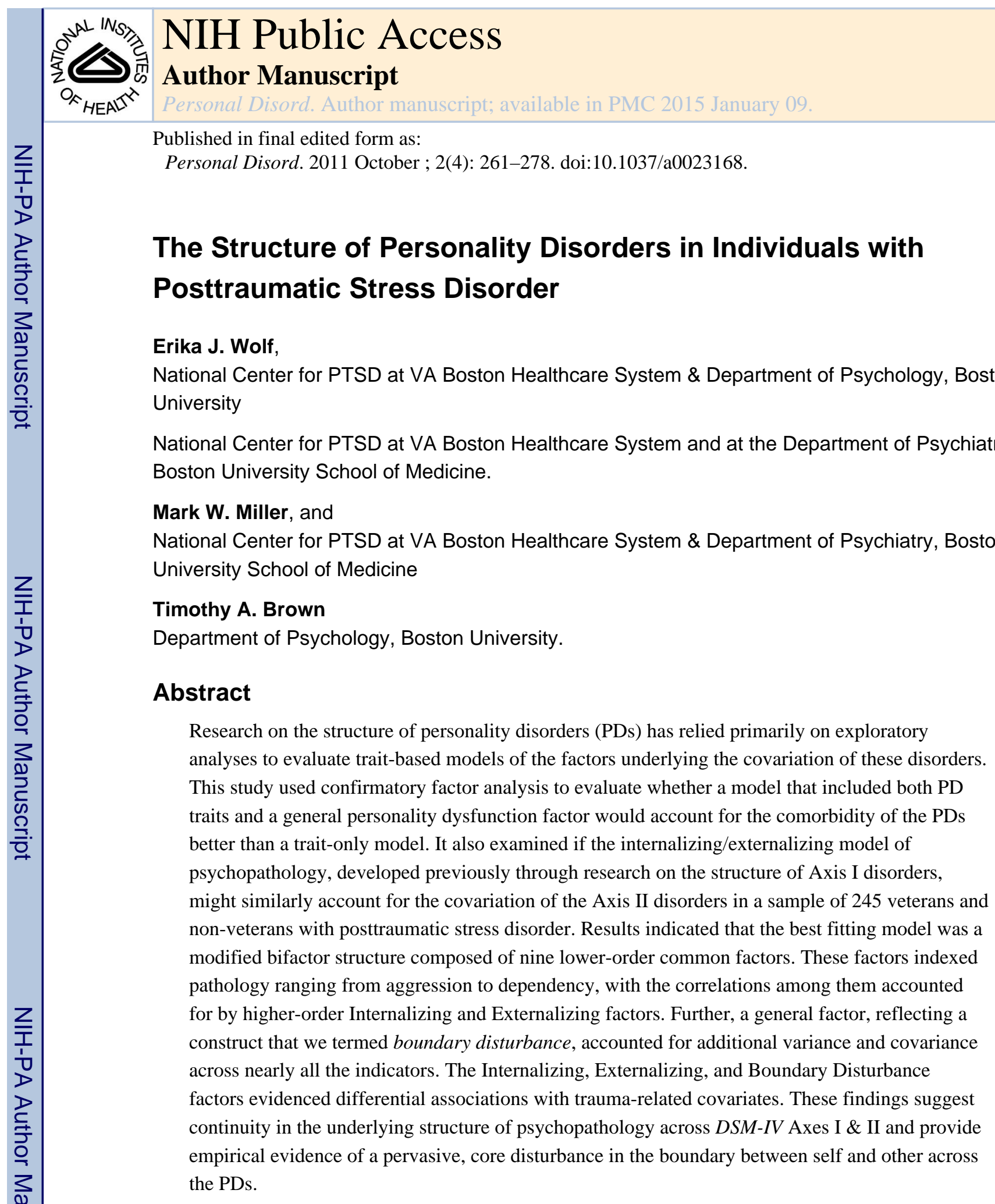

\title{
Keywords
}

personality disorder; internalizing; externalizing; boundary disturbances; posttraumatic stress disorder

\footnotetext{
Correspondence concerning this article should be addressed to Erika J. Wolf, National Center for PTSD (116B-4), VA Boston Healthcare System, 150 South Huntington Avenue, Boston, MA 02130. Erika.Wolf@va.gov..
} 
Adult psychiatric comorbidity (i.e., the co-occurrence of two or more disorders) occurs in replicable and substantive patterns such that the unipolar mood and anxiety disorders form one class of highly related conditions while antisocial personality disorder (ASPD), substance-use disorders, and attention deficit hyperactivity disorder reflect a second, distinct group of highly comorbid diagnoses. Two common factors give rise to this pattern of diagnostic comorbidity: The first, termed internalizing, is conceptualized as the core vulnerability common to the unipolar mood, somatization, and anxiety disorders. The second, termed externalizing, is the common factor underlying the covariation of ASPD and the substance use disorders (Kessler, Chiu, Demler, Merikangas, \& Walters, 2005; Krueger, 1999; Miller, Fogler, Wolf, Kaloupek, \& Keane, 2008; Slade \& Watson, 2006; Vollebergh et al., 2001; Wolf et al., 2010). Aside from a recent examination of the structure of Axis I and II disorders by Markon (2010), ASPD has been the only Diagnostic and Statistical Manual $[D S M]$ personality disorder (PD) included in structural models of internalizing and externalizing comorbidity. Therefore, one aim of this study was to evaluate if this model might similarly account for patterns of diagnostic comorbidity among PDs in a sample of 245 men and women with posttraumatic stress disorder (PTSD). We also sought to examine if a model of the PDs which included both circumscribed PD traits and a pervasive, generalized disturbance in personality functioning would account for the covariation of the Axis II disorders better than a more traditional model composed exclusively of individual traits.

\section{The Structure of Axis II Disorders}

Research on the structure of the PDs has largely converged around evidence that four factors account for the covariation of DSM PD criteria, albeit with still notable variability across the results of such work. These factors are termed "the four As": antisocial, asocial, asthenic (i.e., weak), and anankastic (i.e., obsessive) and are anchored by Cluster B disorders, schizoid PD, avoidant and dependent PDs, and obsessive-compulsive PD, respectively (Mulder \& Joyce, 1997, p. 104). Examination of trait-based measures of personality dysfunction (i.e., an alternative, non-DSM referenced approach to studying the covariation of the PDs) has provided support for four similar factors. These factors have been termed: emotion dysregulation (similar to the asthenic factor), dissocial behavior (akin to the antisocial factor), inhibition (similar to the asocial factor) andcompulsivity (similar to the anakastic factor; Livesley, Jang, \& Vernon, 1998).

These models of PD comorbidity are trait models in that the structure of PDs is conceptualized as arising out of individual differences in circumscribed personality dimensions that underlie both normal-range and disordered personality. However, Livesley and Jang (2005) and Krueger, Skodol, Livesley, Shrout, and Huang (2007) have argued that it is not sufficient to conceptualize PD as simply extreme variants of normal-range personality traits because doing so fails to capture the broader dysfunction that is the most clinically salient aspect of the PDs. They suggested that PDs are comprised of at least two sets of distinct pathological processes: (a) a constellation of maladaptive personality traits and (b) generalized personality dysfunction, that is,characteristics that lead to intra-psychic and/or inter-personal conflicts and impair functioning across domains (Krueger, Skodol, et al, 2007; Livesley \& Jang, 2005). Similarly, the Personality and Personality Disorders DSM- 
$V$ Work Group has included both trait and personality dysfunction criteria in their proposal to reorganize the Axis II disorders. The latter criterion captures "failure to develop a sense of self-identity and the capacity for interpersonal functioning" (American Psychiatric Association [APA], 2010). Despite the potential role that a general personality dysfunction criterion may play in the $D S M-V$, no study to date has included a representation of this construct in factor analytic models of the covariation of the PDs.

The type of structural model that can account for distinct trait factors and a pervasive dysfunction factor is referred to as a bifactor or hierarchical model (cf., Holzinger \& Swineford, 1937; Yung, Thissen, \& McLeod, 1999). In a bifactor model, a general factor influences all of the indicators of a given construct (i.e., the dysfunction factor), and additional, more narrowly defined, factors contribute to subsets of items that are more highly related to one another (i.e., the domain-specific trait factors). The general factor and the specific factors are unrelated to one another (i.e., they contribute to non-overlapping portions of the variance in the indicators). A hierarchical model is distinct from a higherorder model in which the covariance between lower-order factors in the model is accounted for by one or more higher-order factors. To date, no study of the structure of PDs has tested a hierarchical model. The aim of this study was to evaluate this model and to more explicitly define the nature of the primary dysfunction across Axis II.

Another reason why the bifactor model may be particularly applicable to the study of PDs is because personality and PDs exhibit a complex structure (e.g., cross-loadings of indicators on multiple factors), as opposed to a simple one (e.g., each indicator loads on just one factor) and traditional confirmatory factor analysis (CFA) has trouble reflecting this complexity (McCrae, Zonderman, Costa, Bond, \& Paunonen, 1996). Specifying a large number of cross-loadings in a traditional CFA can result in model under-identification and non-convergence. On the other hand, failure to specify necessary cross-loadings in a CFA leads to poor model fit and biased parameter estimates. This is likely one reason why the majority of studies examining the structure of PDs have used exploratory factor analysis (EFA), as every indicator loads on every factor in an EFA. Evaluation of an a priori hypothesized model with differentiation in the item loadings on factors cannot be tested in the EFA framework, nor can other complex, higher-order models. Instead, the best approach to testing such a model is a confirmatory one. If shared variance across all the PDs is due to a single central personality dysfunction factor that is distinct from individual differences in traits, then a bifactor model can resolve the complex structure problem by accounting for the variance that is common across all the PDs on the broad dysfunction factor.

\section{Study Aims and Hypotheses}

The primary aim of this study was to examine the structure of PTSD comorbidity in the Axis II domain using CFA and to evaluate if a model of the internalizing and externalizing structure of primarily Axis I disorders might extend to the PDs. This study also evaluated if a model with both circumscribed PD traits and a pervasive personality dysfunction factor better accounted for the Axis II disorders compared to a more traditional model, comprised only of trait common factors. The top panel of Figure 1 provides a schematic overview of the hypothesized model. It illustrates the modified bifactor model that was tested in which 
one general personality dysfunction factor accounted for covariation across all PD features while additional, more circumscribed, PD trait factors accounted for incremental variance across subsets of more highly related PD features. As shown in Figure 1, higher-order Internalizing and Externalizing factors were specified to account for the correlations among the PD trait factors. It was hypothesized that the construct captured by the general personality dysfunction factor would reflect disturbances in interpersonal relations; a more specific definition of this factor was evaluated empirically by examining the pattern of loadings on this factor. This modified bifactor model was expected to provide significantly better fit to the data than a model without the general common factor. The study also investigated demographic and trauma-related covariates of the major common factors hypothesized to underlie the PDs (i.e., the General PD, Internalizing, and Externalizing factors).

These hypotheses were evaluated in a sample of individuals with PTSD using the DSM-IV PD scales of the Schedule for Nonadaptive and Adaptive Personality (SNAP; Clark, 1996). The study was conducted in a PTSD sample for two primary reasons. First, the disorder is associated with notably high levels (50-80\%) of comorbidity across the PDs (Bollinger, Riggs, Blake, \& Ruzek, 2000; Dunn et al., 2004; Lenzenweger, Lane, Loranger, \& Kessler, 2007; McGlashan et al., 2000; Southwick, Yehuda, \& Giller, 1993) and there is a need to develop a comprehensive yet parsimonious model of this covariation. PTSD that co-occurs with ASPD, for example, is likely to present differently than PTSD that co-occurs with avoidant PD and may be associated with different biological pathways and require different treatment approaches. A model of PTSD-Axis II comorbidity may help to guide the conceptualization of and treatment for this heterogeneity within the PTSD population. Second, PTSD is the only common mental disorder in which disorder onset requires exposure to a well defined environmental pathogen (i.e., a traumatic event). In contrast, the PD criteria include no explicit role for environmental stressors and instead focus on highly stable, temperament-based (i.e., biological) patterns of relating. This study examined of how adverse life events, implicated in the development of PTSD, might also contribute to personality dysfunction. It also provided a preliminary test of how different types of traumatic events might differentially relate to variation in PD pathology.

\section{Method}

\section{Participants}

The sample consisted of 245 veterans and non-veterans who met DSM-IV (APA, 2000) criteria for a current diagnosis of PTSD, as determined by administration of the PTSD Checklist (PCL; Weathers, Litz, Herman, Huska, \& Keane, 1993). They were recruited from a PTSD recruitment database and from flyers posted throughout the hospital. Seventy-three percent of the sample were men $(n=178)$ and $76 \%$ were veterans $(n=186)$. Participants self-reported their race and ethnicity as follows: $66 \%$ White, $24 \%$ Black or African American, 2\% American Indian or Alaskan Native, .4\% Asian, and 7\% selected the "other" response option; in addition, $6 \%$ endorsed their ethnicity as Hispanic or Latino. The mean age of the sample was 47 (range: 19 to 65). Among the veterans in the sample, 36\% had served in the military during the Vietnam War era, 10\% during Operation Desert Storm; 
$10 \%$ in Operation Iraqi Freedom or Operation Enduring Freedom, .4\% in the Korean War era, and $20 \%$ during other intervals. Thirty-two percent of the sample endorsed combat trauma as the Criterion A event they completed the PCL in reference to, $16 \%$ endorsed physical assault, $12 \%$ endorsed childhood sexual trauma, $11 \%$ endorsed the sudden death of a friend or loved one, 9\% endorsed sexual trauma as an adult only, $7 \%$ endorsed non-sexual childhood trauma, and 5\% endorsed a motor vehicle accident as the index trauma. Several other types of trauma were endorsed by the sample (e.g., natural disaster, serious accident), each occurring in less than $2 \%$ of the sample.

Procedure

The telephone eligibility screen began with a brief assessment of trauma exposure by asking a question drawn from the United States Department of Veterans Affairs (VA) PTSD screen. Participants then provided a short description of the trauma to determine that the event met DSM-IV PTSD Criterion A. The PCL (Weathers et al., 1993), a reliable and valid measure of PTSD, was then administered over the telephone and participants who met DSM-IV criteria for current PTSD (see below) were invited to participate in the study. Participants completed self-report measures, including a second PTSD assessment using a paper and pencil administration of the PCL, in one 2-3 hour in-person session and were compensated for their time and effort. The study was approved by all relevant institutional review boards.

\section{Measures}

The Traumatic Life Events Questionnaire (TLEQ; Kubany et al., 2000)—The TLEQ is a self-report measure that assesses exposure to 21 different traumatic events that meet the DSM-IV PTSD Criterion A1 definition for a traumatic event. For each traumatic event that is endorsed, a follow-up question assesses DSM-IV PTSD Criterion A2 by asking if the individual experienced intense fear, helplessness, or horror in response to the event. The number of times the event was experienced is also assessed on a 7-point scale ranging from "never" to "more than five times." The TLEQ has been shown to have good test-retest reliability over a two-week interval (mean kappa $=.63$, mean percent agreement $=86 \%$ ), excellent content and convergent validity with interview-based measures of trauma exposure (mean percent agreement $=92 \%$ ) and good predictive validity (higher scores on the TLEQ associated with PTSD status; Kubany et al., 2000).

PCL (Weathers et al., 1993)-The PCL is a 17-item measure designed to assess the $D S M-I V$ criteria of PTSD. Respondents rated the extent to which they have been bothered by symptoms over the past month on a 5-point scale ranging from 1 ("not at all") to 5

("extremely"). The PCL has excellent concurrent validity with interview-based measures of PTSD ( $r=.79$ - .93; Blanchard, Jones-Alexander, Buckley, \& Forneris, 1996; Keen, Kutter, Niles, \& Krinsley, 2008) and test-retest reliability $(r=.96$; Keen et al., 2008; Weathers et al., 1993). Per DSM-IV PTSD criteria, probable PTSD diagnosis was defined as endorsement of at least one PTSD Criterion B symptom, three Criterion C symptoms, and two Criterion D symptoms at a symptom rating of 3 ("moderate") or higher on the 1-5 scale. This scoring rule for the PCL has been endorsed by Weathers et al. (1993) and evidenced better diagnostic efficiency relative to the PCL cut-off scoring approach (Keen et al., 2008). Further, this scoring rule is more appropriate for determining PTSD diagnostic status in 
mixed samples (e.g., veterans and non-veterans, variation in the index trauma) as compared to the cut-off score approach because optimal cut-off values for the diagnosis differ as a function of population.

SNAP (Clark, 1996, 2003)—The SNAP is a 371-item factor-analytically derived truefalse inventory of personality and personality dysfunction. The measure includes 12 trait and 3 temperament scales that assess maladaptive personality styles that are not referenced to specific $D S M$ criteria. The 12 trait scales were developed with the aim of identifying the core, unidimensional, maladaptive personality traits that cut across the DSM PD criteria rather than measuring $D S M$-derived criteria directly. The measure also includes 6 validity scales. When administered with the 36-item SNAP Supplement (Clark, 2003), the SNAP also yields 10 PD scales with diagnostic and dimensional scores referenced to the DSM-IV Axis II criteria. Items in the PD scales were created rationally and refined empirically and include item overlap with the trait scales. Results from the SNAP validation study suggest that the PD scales are internally consistent (median alpha in clinical sample $=.79$; Clark, 1996) and evidence adequate convergent validity with interview-based measures of PD (mean correlation with structured interview in normative sample $=.58$; Clark, 1996). This study did not evaluate the trait scales as the goal was to examine the structure of disorderspecific DSM-referenced PD criteria in order to evaluate findings in the context of the DSM conceptualization of PD. Given this, the 10 DSM-IV dimensional PD scores were evaluated, however, because these summary scores are multidimensional and involve item overlap across the scales, each PD scale was subdivided into smaller, more unidimensional parcels, as detailed below.

\section{Data Analyses}

Rationale for creation of SNAP PD parcels-The SNAP PD scales are each multidimensional, arguably reflecting the multidimensionality of the Axis II criteria in the $D S M-I V$. Such multidimensionality is problematic because scales that are not unidimensional will evidence diverse patterns of association when subjected to factor analysis (e.g., cross-loadings and residual covariances) and the use of single summary scores for each PD scale will obscure the underlying reason for these multivariate associations. Furthermore, cross-scale item overlap on the scales renders it impossible to determine if scales correlate with one another for substantive reasons or if the correlation is simply a reflection of the shared items. To address these problems, the scoring of the SNAP PD scales was revised by eliminating item overlap across the scales and creating more unidimensional parcels, as detailed below.

Creation of SNAP PD parcels-A rational/theoretical approach was followed for creating parcels for each PD scale and each was then empirically evaluated and further refined. Parcels for each PD scale were created by taking the mean of items from two or more $D S M-I V$ criteria that appeared to reflect a shared construct. In creating the parcels, only items from the same parent PD scale were combined. Individual items from a specific PD criterion that were not a good marker of the parcel (based on item content) were removed from that one and added to a different parcel from the same parent PD scale. In addition, items that overlapped across PD scales were removed from one of the scales so 
that each item contributed only to one parcel. The decision regarding which scale to remove the overlapping item from was based on the content of the item and its relative fidelity for the diagnoses overall, and the parcels, specifically. Every effort was made to balance dropped items across scales so that no scale would lose a disproportionate number of items and so that each PD criteria represented by the SNAP would be incorporated into a parcel. There was only one instance in which all the items from a given criterion were eliminated.

This rational approach was further refined by empirical examination using classical test construction methods, with the goal of creating unidimensional and clinically meaningful subscales that faithfully represented the content domain of each SNAP PD scale.

Specifically, the internal consistency (Cronbach's alpha) of each parcel was examined, as was the correlation between each individual item and the parcel total score. Parcels with alpha values less than .50 were rejected and refined; items that correlated with the parcel total less than $r=.30$ were removed from that parcel and either placed on a parcel from the same PD that they correlated more strongly with or removed from the scale altogether. Across all the parcels, only eight out of 249 items (3\%) were completely eliminated for this reason. Finally, the correlations between parcels from the same PD were examined and parcels that were highly correlated with one another were either combined or further refined. This approach resulted in 35 parcels (see supplemental Table 1). The parcel name, alpha coefficient, and the mean correlation between each item and the parcel total are shown in supplemental Table 1, as is an example of the item content of the parcel. Item scoring for the parcels is available from the first author.

Analytic approach-All structural analyses were conducted with the Mplus 5.1 (Muthén, \& Muthén, 1998-2009) statistical modeling software. First, exploratory structural equation modeling (ESEM) was conducted to guide the development of the lower-order PD trait structure of the SNAP parcels, as no prior study has evaluated the trait factor structure of the SNAP DSM-IV PD scales. ESEM is a new analytic approach that combines elements of EFA and CFA. Like EFA, it is appropriate in the analysis of items with a complex structure (e.g., multiple cross-loadings) and allows for model identification by fixing factor variances to 1 and rotating the factors (Asparouhov \& Muthén, 2009; Marsh et al., 2009, Muthén \& Muthén, 1998-2009). Also like EFA, it can produce exploratory results for a number of possible factor solutions specified by the user. Like CFA, however, one can also impose a priori associations among the data, such as adding residual covariances, covariates, and structural paths.

Results from the ESEM were used to inform the CFA. Specifically, the results of the ESEM were used to help guide the specification of the relationship between the parcels and the lower-order, circumscribed PD trait factors. Higher-order Internalizing and Externalizing factors were specified to account for the covariation among the lower-order trait factors. A general factor, reflecting the broad personality dysfunction construct, with loadings on all of the SNAP parcels was also specified; this factor had no association with any other factors in the model. Thus this model included elements of both higher-order (the lower-order trait and higher-order Internalizing and Externalizing factors) and hierarchical (the general factor) models; for this reason, it is referred to here as a "modified bifactor model." The necessity of specific loadings and structural paths were then examined empirically and refined in a 
model testing sequence. Given that some of the parcels were means of only two or three items and their variance was limited, the CFA was conducted with a mean and variance adjusted maximum-likelihood estimator (MLMV). The ESEM was conducted with the maximum likelihood (ML) estimator as the MLMV estimator is not currently available for use with ESEM. Goodness of fit was determined with statistics from the absolute fit, parsimony, and comparative fit classes of indices, namely: chi-square, standardized root mean square residual (RMR), root mean square error of approximation (RMSEA), TuckerLewis index (TLI), and comparative fit index (CFI). As suggested by Hu and Bentler (1999), RMSEA values $<.06$, RMR values $<.08$, and TLI and CFI values $\geq .95$ were considered as indicators of good fit. These fit statistics were evaluated as a whole, such that a single value that fell just outside of the acceptable range would not necessarily indicate poor model fit if other statistics were consistent with good fit (Brown, 2006).

\section{Results}

\section{Examination of PD Trait Factors with ESEM}

Using ESEM, factor solutions were obtained for 2 to 6 factors with geomin (i.e., oblique) rotation of the factors. The fit of the models is shown in Table 1. None of the models were consistent with good model fit, however, the fit statistics for the 4, 5, and 6 factor solutions were notably improved relative to the others and began to approach acceptable fit. The scree plot suggested four to five factors, thus the interpretability of the four and five factor solutions was further examined (detailed results of this intermediate analysis are available from the first author). The first factor in the four factor model was marked by parcels reflecting aggressive and impulsive behavior and negatively, by traits such as constraint and conscientiousness. The second factor was marked primarily by parcels associated with selfand other-alienation. The third factor appeared to reflect the dimension of exhibitionism and was negatively associated with traits related to introversion and aloofness. The fourth factor was marked by parcels reflecting insecurity, stress reactions, and dependence on others. The five factor model differed from the four factor model in that traits related to impulsivity versus aggression were split on two separate factors; this distinction appeared conceptually meaningful, thus the five factor model was retained for further analysis.

The five factor ESEM was marked by a high number of small to moderate in magnitude, yet statistically significant, cross-loadings. In addition, the results yielded a large number of modification indices, reflecting covariance among the parcels that was not represented in the model. Examination of these modification indices revealed only one that appeared to reflect a method effect. Specifically, approximately three-quarters of an item from the borderlineinsecure attachment parcel was embedded, word-for-word, in an item from the dependenthelplessness parcel. Thus, in an additional ESEM, a five factor solution with one correlated error reflecting this method effect was specified. The fit of this model was largely acceptable (see Table 1), although the TLI value was below the cut-off and the modification indices continued to suggest underrepresented parcel covariance that were not due to discernable method effects. 


\section{Development of Modified Bifactor CFA}

The results of the ESEM suggested the presence of five common PD trait factors and one correlated error between two parcels; these factors were specified in the CFA, with loadings that were statistically significant and clinically substantive in the ESEM included as indicators of these factors. The modification indices from the ESEM were also used to develop additional trait factors in the CFA. Specifically, a closer examination of these modification indices suggested that four of them might be due to clinically substantive associations among the PD parcels that were not represented by the model (in other words, the model was under-factored). For example, the schizotypal-mistrust and paranoid-mistrust parcels generated a large modification index, suggesting the possibility of a mistrust common factor. Thus, in specifying the PD trait factors in the CFA, these four modification indices were used to develop four additional trait factors. To do so, the two parcels that produced the modification index were set as indicators of a common factor and at least one additional parcel was set to index the factor as well; this third loading was specified based on clinical and theoretical rationale and refined as needed. Only theoretically and/or clinically justifiable factors were pursued in this phase of model development. The bottom panel of Figure 1 provides a general schematic of this approach to developing the PD trait factors in the CFA. The four additional factors that were developed in this phase of the analysis reflected emotional dysregulation, odd/peculiar behavior, mistrust, and achievement/compulsivity.

These four factors were combined with the five factors that emerged directly from the results of the ESEM to yield nine PD trait common factors, which were specified to load on two higher-order factors: Internalizing and Externalizing. Specifically, the Aggression, Emotional Dysregulation, and Impulsivity factors were specified as indicators of the higherorder Externalizing factor and the Social Alienation, Mistrust, Achievement/Compulsivity, Odd/Peculiar, and Dependence/Insecure Attachments factors were specified as indicators of the higher-order Internalizing factor. The Exhibitionism factor was initially not specified to load on either Internalizing or Externalizing because in preliminary analyses it did not appear to evidence a robust association with the other PD trait factors in the model; instead, its associations with the two higher-order factors were explored through a model testing sequence (see below). In addition to the nine PD trait factors and the two higher-order Internalizing and Externalizing factors, a General PD factor was specified with loadings from all 35 PD parcels (i.e., the hierarchical component of the model). The correlation between this General PD factor and all other factors in the model was set to 0 . The only correlated residual specified in these models was that between dependent-helplessness and borderline-insecure attachment, as described previously. All indicators in this model loaded significantly on their respective trait factors and the model yielded no modification indices that were justifiable for use in the development of additional factors. The fit statistics for this model (Model 1), as shown in the first row of Table 2, were consistent with good model fit.

Refinement of general factor associations-Next, a nested-model design was used to examine the necessity of non-significant loadings on the General PD factor. Specifically, paranoid-aggression, antisocial-aggression, antisocial-impulsivity, borderline-dissociation, avoidant-fear of rejection, and obsessive-compulsive-work ethic evidenced non-significant 
loadings on the general factor. Of these, five parcels evidenced loadings on the PD trait factors > .75, and the remaining parcel (obsessive-compulsive-work ethic) showed a PD trait factor loading of .65. Given these strong trait factor loadings, one potential reason for the lack of association between these parcels and the General PD factor is that there was simply no true score variance remaining after accounting for these primary-level associations. Thus, these six paths were eliminated in the next model and the fit of this nested model was compared with the parent model. This analysis revealed that eliminating these paths did not damage model fit (see Model 2 in Table 2), thus Model 2 was retained for further refinement.

Refinement of structural associations-Next, the relationships between the Exhibitionism factor and the higher-order Internalizing and Externalizing factors were tested. To do so, the Exhibitionism factor was first set to indicate both Internalizing and Externalizing. This model is not nested within the best fitting model previously obtained, as this model adds, rather than removes, paths (but see model comparison, below). This model fit the data well (see Model 3 in Table 2) and the Exhibitionism factor evidenced a significant loading on latent Internalizing (completely standardized loading $=-.29, p<.01$ ), but not on latent Externalizing (completely standardized loading $=.07, p=.48$ ). Model 3 was then compared with a nested model in which the path from Externalizing to Exhibitionism was removed (Model 4). This analysis revealed that removing this path did not damage model fit (see Model 4 in Table 2). The necessity of the pathway from Internalizing to Exhibitionism was evaluated by eliminating it and comparing the fit of this model (Model 5) with the fit of Model 4, in which this path was estimated. This CFA revealed that eliminating this path damaged model fit (see Model 5 in Table 2), thus Model 4 was retained as the best fitting model.

Figure 2 shows the completely standardized coefficients of the structural paths in the model. Factor loadings are shown in Table 3 , as is indicator error. All paths were statistically significant at the $p<.05$ level (most at the $p<.001$ level) with the exception of the path from the General PD factor to the borderline-self harm parcel (loading $=.11, p=.11$ ) and to the histrionic-emotional expression parcel (loading $=.10, p=.06$ ). These two loadings were statistically significant in Model 1, prior to dropping all non-significant paths from the General PD factor to the indicators. Given this, and given that the $p$-values associated with these paths approached significance, these paths were retained.

Comparison with PD trait only model—Finally, the fit of the best-fitting model (Model 4 in Table 2) was compared with the fit of a nested model which was identical except that the General PD factor was eliminated (i.e., a higher-order model with no hierarchical component). This evaluated if a model which incorporated both PD traits and general personality dysfunction fit better than a more traditional trait only model. The trait only model fit the data poorly, resulted in a Heywood case (i.e., a parameter estimate that was out-of-range) in the estimated residual of one parcel, and significantly degraded model fit compared to the modified bifactor model (see Model 6 in Table 2). 


\section{Trauma and Demographic Covariates}

Next, structural equation modeling (SEM) was used to explore demographic and traumarelated covariates of the Internalizing, Externalizing, and General PD factors. Specifically, the best fitting model from the previous analyses (Model 4 in Table 2) was specified and age, gender, minority status, exposure to childhood sexual trauma (yes/no), exposure to combat trauma (yes/no), and the number of different types of trauma endorsed on the TLEQ were simultaneously regressed on the Internalizing, Externalizing, and General PD factors. Results revealed that: (a) the General factor was predicted by age (path $=-.27, p<.001$ ), and exposure to childhood sexual trauma (path $=.15, p=.038$ ); (b) the Internalizing factor was predicted by gender (males coded as reference group, path $=-.14, p=.032$ ), and the number of different traumas one was exposed to (path $=.36, p<.001$ ), and (c) the Externalizing factor was predicted by gender (path $=-.34, p<.001$ ), age (path $=-.13, p=$. 005 ), number of different traumas (path $=.37, p<.001$ ), and exposure to combat (path $=.13$, $p=.052)$.

In a follow-up SEM, the association between PTSD severity and the Internalizing, Externalizing, and General PD factors was examined. Latent PTSD was specified as a single indicator of summary scores on the PCL, corrected for measurement error based on examination of alpha in this dataset (alpha $=.92)$, and set to correlate with these common factors from the final model. This analysis suggested that PTSD severity was significantly correlated with both Internalizing $(r=.53, p<.001)$ and Externalizing $(r=.40, p<.001)$ but not with the General PD factor $(r=.02, p=.74)$.

\section{Discussion}

This study evaluated whether a modified bifactor model of the structure of PDs that included representations of both circumscribed traits and generalized dysfunction in self-other relationships might account for the covariation of PDs better than a traditional model comprised of only specific traits. It also examined if the internalizing/externalizing model, derived from research on the comorbidity of Axis I disorders, would similarly account for covariation amongst the PDs in a sample of individuals with PTSD. Following a series of preliminary exploratory analyses to identify the specific traits underlying the SNAP PD scales, a series of CFAs was conducted which ultimately yielded support for a combined hierarchical/higher-order, dimensional model. The model was defined by nine lower-order PD traits that loaded on two higher-order factors resembling internalizing and externalizing. The model also included a general factor, hereafter referred to as Boundary Disturbance, which exerted a pervasive effect across nearly all of the PD parcels.

\section{The Lower-Order PD Traits and Higher-Order Dimensions}

The nine lower-order factors identified in this study represent the primary PD traits of the SNAP PD scales. They cut across PD diagnoses, reflect individual differences in tendencies towards specific forms of pathological behavior, emotion, and cognition, and bear close resemblance to factors identified in prior studies of the structure of Axis II disorders. The first three factors, Aggression, Emotional Dysregulation, and Impulsivity, are facets of externalizing psychopathology. Aggression was defined by parcels reflecting pure angry and 
antagonistic behavior as well as an obsessive-compulsive PD parcel reflecting rigidity and stubbornness. The latter (somewhat counterintuitive) finding may provide an explanation for prior reports of covariation between antisocial PD and obsessive-compulsive PD (e.g., see tetrachoric correlation of .45 between these disorders in the National Comorbidity Survey Replication; Lenzenweger et al., 2007). The second factor, Emotional Dysregulation, reflected lability that was largely specific to the domains of anger and arousal making this factor more specific to disinhibited negative emotional arousal, as opposed to prior work which identified a broader Emotional Dyregulation factor (i.e., Livesley et al., 1998) that included greater contribution of generalized distress and negative emotional valence to the construct. The third factor, Impulsivity, accounted for common features across socially deviant, reckless, and sensation-seeking parcels from the antisocial and borderline scales and was negatively associated with perfectionism and moral inflexibility. SEM analyses showed that significant predictors of the Externalizing factor were gender (men had higher means on the latent variable), age (an inverse association), greater exposure to trauma, and exposure to combat trauma, specifically. This is consistent with prior research on the association between gender (Kramer, Krueger, \& Hicks, 2008), age (Hurt \& Oltmanns, 2002; Littlefield, Sher, \& Wood, 2009) and externalizing psychopathology. The specificity of the relationship between combat exposure and externalizing was unexpected, however, and may be a function of the large percentage of male veterans in this sample.

The remaining lower-order traits identified in this study involved tendencies towards alienation, agitation, withdrawal, and anxiety and loaded on a second higher-order factor reflecting internalizing. The composition of three of these factors, Social Alienation, Exhibitionism, and Dependency/Insecure Attachments, and their pattern of higher-order factor loadings was consistent with prior work on the internalizing construct. They captured social isolation, the lack of internalized distress, and personal insecurities, respectively. The remaining three traits, Odd/Peculiar, Mistrust, and Compulsivity/Achievement, reflected somewhat broader manifestations of internalizing compared to prior studies of Axis I disorders, but may represent important facets of the construct. Specifically, the Odd/Peculiar factor was marked by tendencies towards cognitive and affective aberrations--phenomena associated with social isolation and psychological distress. A similar factor, labeled Thought Disorder, was identified by Markon (2010) in a recent examination of the structure of Axis I and II disorders and it too evidenced a strong association $(r=.72)$ with the Internalizing factor identified in that study. Taken together, results of these two studies suggest that the Odd/Peculiar factor may reflect an important, yet previously unexplored, aspect of the internalizing dimension. Another novel facet of internalizing was Mistrust. Items accounted for by this factor reflected anxiety and perseveration about potential harm and the trustworthiness of others. While the content of this type of rumination differs from that associated with a primary anxiety disorder, the processes and consequences are similar: social avoidance and detachment. Finally, the Compulsivity/Achievement factor was defined by a tendency towards a rigid and narrow adherence to rules, and a single-minded focus on productivity and goals at the expense of social relationships; when goals are unmet or tasks incomplete, frustration and agitation ensue. Together, the Odd/Peculiar, Mistrust, and Compulsivity/Achievement factors captured clinically meaningful behavioral manifestations of the internalizing spectrum that have to date, gone unexplored, but may reflect internalized 
psychiatric distress in the domain of maladaptive personality traits. Men scored higher on latent Internalizing compared to women, in contrast to prior work suggesting that women are likely to have higher latent means on Internalizing (Kramer et al., 2008). Greater trauma exposure was also associated with higher means on this factor, suggesting a dose-response relationship between trauma exposure and trait-based internalizing psychopathology.

\section{Hierarchical Common PD Factor: Boundary Disturbance}

This study is the first to identify the presence of a hierarchical factor that accounts for the general covariance across all the PDs and across nearly all the specific PD features represented in the SNAP, but is statistically and conceptually distinct from the specific trait factors. This factor was termed Boundary Disturbance and defined as impairment in the ability to self-regulate drives for affiliation and agency, which leads to violations in social mores and disrupted interpersonal relations. This definition was developed by studying the pattern of factor loadings on the General PD factor and theorizing about the common elements of Axis II pathology. The factor was marked most strongly by PD parcels reflecting attention-seeking, dramatic, and manipulative characteristics and negatively by PD parcels reflecting social isolation, aloofness, and fears of personal inadequacy. Sample items characteristic of this factor included: "I like to show off," "I dress to attract sexual attention," and "I deserve to be admired." Items such as "I don't particularly like spending time with people," and "I feel so inadequate when meeting new people that I usually just keep to myself" contributed to parcels with strong negative loadings on this factor. Traits such as social deviance, perfectionism, antagonistic relationships, emotional reactivity, and an excessive need for nurturance were more modestly related to this factor. This suggests a bipolar dimension of self-relationship disturbance defined, on one pole, by extreme affiliative and agentic tendencies (i.e., a desire for social connection, to be liked, and in control) that are manifested in overbearing, antagonistic, or dramatic behavior and, on the other pole, by asocial, isolative tendencies manifested in self-doubt, and stilted, awkward interpersonal behavior. Both poles reflect problems in conforming to societal rules and expectations for managing relationships.

Though the Boundary Disturbance and PD trait factors are conceptually and empirically distinct, their interaction may give rise to specific manifestations of personality dysfunction. For example, for those with an underlying trait disposition towards exhibitionism, difficulty modulating boundaries may result in intrusive, histrionic-type behavior. In contrast, for those with an underlying trait disposition towards dependency, impaired regulation of boundaries may result in insecure attachments and associated dependent behavior. Unlike the specific traits, which have a basis in temperament, boundary disturbance may reflect impairment in the ability to access and manipulate learned schemas about social interaction, leading to dsyregulated interpersonal behavior. Although these data don't address the mechanism underlying this impairment, one possibility is that boundary disturbance is a function of stress-related deficits in executive functioning, as prior work suggests that PDs share genetic variance with problems in this area of cognitive function (Coolidge, Thede, \& Jang, 2004). The Boundary Disturbance factor identified in this study should be of particular interest to theorists who have asserted that current models of PD are missing a core element of what defines PDs and makes working with individuals with PDs particularly challenging 
(i.e., Krueger, Skodol et al., 2007; Livesley \& Jang, 2005; Shedler \& Westen, 2004a). It is also consistent with a range of both early and contemporary theories on the nature of PDs, supporting the central importance of self-other instability across these disorders (see Lenzenweger \& Clarkin, 2005 for a review of PD theory).

SEM analyses showed that, of the trauma variables, only childhood sexual trauma was a significant predictor of Boundary Disturbance. This is consistent with theory (Herman, 1992) and research (Cloitre et al., 2009) regarding the long term effects of childhood sexual trauma on self-regulation and interpersonal styles. Taken together with the pattern of trauma covariates for the Internalizing and Externalizing factors, this suggests that different types of trauma may exert differential effects in the development of psychopathology and selfregulatory processes. That is, while greater trauma exposure may be a general risk factor for maladaptive personality traits, childhood sexual trauma may have a more specific effect on self-other relational styles. However, as this study is cross-sectional in design, these results cannot address the causal or etiologic role of trauma exposure on these constructs. Finally, the finding that PTSD severity was associated with the Internalizing and Externalizing factors but not with the Boundary Disturbance factor suggests that the General PD factor is distinct from psychopathology symptoms, which would be expected to covary with PTSD severity. This provides further evidence of the distinction between PD traits and PD dysfunction.

\section{Study Limitations and Strengths}

The findings of this study are limited by several methodological issues. First, the study included only self-report measures, raising questions about the validity of the diagnoses and symptoms assessed. There was no way to determine the number of participants who met full diagnostic criteria for a PD and this bears on the relevance of these structural findings for the PD population. However, this concern is ameliorated by evidence for the dimensionality of the Axis II disorders (Widiger \& Simonsen, 2005), as subthreshold features of PD are thought to reflect the same construct as diagnostic levels of these disorders. Along with concern about the arbitrary nature of the diagnostic cut-points in the DSM, this implies that use of a sample that may include subthreshold cases still has relevance for the broader PD population. Second, this dataset did not permit a direct test of the degree to which the Axis I versus Axis II-derived internalizing/externalizing dimensions overlap (doing so would require an additional assessment of Axis I disorders in this sample). Nevertheless, the features shared across the PD traits that loaded on Internalizing (i.e., social withdrawal, anxiety, and distress) and Externalizing (i.e., impulsivity, aggression, and deviant behavior) in this study are consistent with prior work on these domains. The lack of additional Axis I measures also made it impossible to characterize the sample with respect to such psychopathology. Third, there were no additional measures available to evaluate the construct validity of the factors identified in this study. Fourth, as discussed previously, the study was based on a convenience sample of mostly male veterans with PTSD, raising the possibility that the results might not generalize to non-veterans, women, or to PD samples without PTSD and/or significant trauma histories. That said, it seems unlikely that the basic structure of PD would be dependent on the sample demographic as structural analyses of psychopathology are generally robust across populations (see Krueger, Chentsova-Dutton, 
Markon, Goldberg, \& Ormel, 2003). Further, studies of primary PD samples often report high rates of comorbid PTSD (Lenzenweger et al., 2007; McGlashan et al., 2000), suggesting the relevance and external validity of studying PD in a PTSD sample. Although PD comorbidity is not unique to PTSD, the use of a PTSD sample capitalized on the high rates of PD pathology associated with the diagnosis and provided the opportunity to develop a conceptual model for the heterogeneity of posttraumatic psychopathology. Further evaluation of this model in samples drawn from other PD populations and using other indicators of PD will be necessary to evaluate the validity and utility of this model.

An additional point to consider is that, in the final model, two of the four fit indices fell just outside the range of acceptable model fit. Specifically, the CFI and TLI values of this model were .90 while values of .95 and above are the current cut-point for evaluating model fit (Hu $\&$ Bentler, 1999). Although this represents a source of concern, there are several reasons why the model should not be rejected based on these values. First, it has generally been accepted that CFI and TLI values of .90 and above are suggestive of "reasonable" model fit (King et al., 2000, p. 628) and this convention is evident in current applied research (e.g., Elhai et al., 2009). Second, Marsh, Hau, \& Wen, (2004) cautioned against a stringent interpretation of Hu and Bentler's (1999) cut-off guidelines, noted that these cut-off values are often difficult to obtain in applied research, and raised questions about the general approach used by $\mathrm{Hu}$ and Bentler to obtain these decision rules. Finally, in this study, the RMSEA and RMR values were consistent with good model fit, and there were no indicators of localized model strain (e.g., in the standardized residuals or modification indices).

Lastly, as is the case with any SEM study, there are other possible models of the latent structure of Axis II disorders that were not examined that could provide equivalent or improved model fit. Evidence for the validity of this model will require replication in other samples, with different measures and demographic characteristics, and continued comparison between this and competing models. These concerns are arguably offset by the strengths of this study, which include: (a) the first empirical examination of a model of Axis II with both PD traits and general PD dysfunction represented; (b) the evaluation of the relevance of the internalizing and externalizing common factors to Axis II; (c) the use of a confirmatory analytic approach in a literature saturated with exploratory modeling; and (d) the use of an empirical approach to evaluate and further define the nature of general PD dysfunction.

\section{Implications for DSM and Conclusion}

In total, the results of this study underscore the poor discriminant validity and heterogeneity of the Axis II disorders, as currently organized in the DSM-IV. Specifically, in this study, parcels from the same diagnosis evidenced varied patterns of associations rather than loading on a single underlying factor. This pattern of results suggests a lack of coherency and validity to the organization of the DSM-IV Axis II disorders, a problem which is likely to hamper the study of the etiology, pathophysiology, and treatment of these disorders.

Noting the problem of disorder overlap among the PDs, many experts in this area, although not all (see Shedler \& Westen, 2004a, 2004b), have begun to support a reorganization of the Axis II disorders based on the maladaptive poles of normal range models of personality, 
with such proposals based primarily on exploratory trait-only models (e.g., Livesley et al., 1998; Widiger \& Mullins-Sweatt, 2008; Widiger \& Simonsen, 2005). As described on the $D S M-V$ website, the proposed revisions to the PD criteria include both PD traits and general PD dysfunction (APA, 2010), but it appears that, to date, no study has empirically evaluated this type of structure. The results of this study suggest that it is possible to test this complex structure and to move beyond the exploratory analyses that have characterized this line of work. This study is the first to demonstrate empirically that both PD trait and dysfunction constructs are important for understanding the comorbidity of the PDs. Inclusion of a Boundary Disturbance, or related personality dysfunction criteria set as part of the DSM definition of PDs might help to quantify the severity of psychopathology and characterize the types of behaviors that may be primary targets of treatment.

A final point for consideration in $D S M-V$ is the broader role of the internalizing and externalizing dimensions. Widiger and Simonsen (2005) proposed a hierarchical and dimensional model of Axis II disorders that included superordinate internalizing and externalizing factors which were thought to subsume lower-order PD and personality traits. These results suggest that the internalizing and externalizing common factors identified in prior studies of Axis I disorders may extend into the realm of Axis II psychopathology, accounting for much of the covariation of the PDs as well. This suggests that as revisions to the DSM are pursued based on the internalizing and externalizing structure of the Axis I disorders (Goldberg, Krueger, \& Andrews, 2009; Krueger \& South, 2009; Watson, 2005), a similar revision should be considered for the Axis II disorders. To the extent that the same dimensions underlie the Axis I and II disorders, this may suggest that: (1) personality pathology is not limited to the PDs and is important for the assessment, conceptualization, and treatment of both Axis I and II disorders (see also Brown \& Barlow, 2009; Krueger, Skodol et al., 2007); and (2) that the same genetic and environmental factors may broadly increase the risk for internalizing versus externalizing disorders across the Axes. One possibility raised by results of this study is that the primary distinction between PDs and Axis I disorders is the presence or absence of boundary disturbances, and this characteristic, rather than individual differences in personality traits, may be used to denote the pervasiveness and intractability of personality dysfunction.

\section{Supplementary Material}

Refer to Web version on PubMed Central for supplementary material.

\section{Acknowledgments}

Funding for this study was provided by a National Institute of Mental Health award 5F31MH074267 to Erika J. Wolf, and by a VA Merit Review Award to Dr. Miller.

We would like to thank Christopher J. Patrick, Ph.D., and Robert F. Krueger, Ph.D., for their guidance on the data analytic strategy and the interpretation of the results of this study.

\section{References}

American Psychiatric Association. Diagnostic and statistical manual of mental disorders. 4th ed Text Revision. Author; Washington, DC: 2000. 
American Psychiatric Association. [March 15, 2010] Personality and Personality Disorders. 2010. from http://www.dsm5.org/ProposedRevisions/Pages/PersonalityandPersonalityDisorders.aspx

Asparouhov T, Muthén B. Exploratory structural equation modeling. Structural Equation Modeling. 2009; 16:397-438.

Blanchard EB, Jones-Alexander J, Buckley TC, Forneris CA. Psychometric properties of the PTSD Checklist. Behaviour Research and Therapy. 1996; 34:669-673. [PubMed: 8870294]

Bollinger AR, Riggs DS, Blake DD, Ruzek JI. Prevalence of personality disorders among combat veterans with posttraumatic stress disorder. Journal of Traumatic Stress. 2000; 13:255-270. [PubMed: 10838674]

Brown, TA. Confirmatory factor analysis for applied research. Guilford Press; New York: 2006. Introduction to CFA.; p. 40-102.

Brown TA, Barlow DH. A proposal for a dimensional classification system based on the shared features of the DSM-IV anxiety and mood disorders: Implications for assessment and treatment. Psychological Assessment. 2009; 21:256-271. [PubMed: 19719339]

Clark, LA. Schedule for Nonadaptive and Adaptive Personality. Manual for administration, scoring, and interpretation. University of Minnesota Press; Minneapolis: 1996.

Clark LA. Schedule for Nonadaptive and Adaptive Personality-2TM (SNAP-2)TM. 2003Used by permission of the University of Minnesota Press Unpublished Test Booklet. Copyright (C 2003 the Regents of the University of Minnesota. All rights reserved.

Cloitre M, Stolbach BC, Herman JL, van der Kolk B, Pynoos R, Wang J, et al. A developmental approach to complex PTSD: Childhood and adult cumulative trauma as predictors of symptom complexity. Journal of Traumatic Stress. 2009; 22:399-408. [PubMed: 19795402]

Coolidge FL, Thede LL, Jang KL. Are personality disorders psychological manifestations of executive function deficits? Bivariate heritability evidence from a twin study. Behavior Genetics. 2004; 34:75-83. [PubMed: 14739698]

Dunn NJ, Yanasak E, Schillaci J, Simotas S, Rehm L, Souchek J, Menke T, Ashton C, Hamilton JD. Personality disorders in veterans with posttraumatic stress disorder and depression. Journal of Traumatic Stress. 2004; 17:75-82. [PubMed: 15027797]

Elhai JD, Engdahl RM, Palmieri PA, Naifeh JA, Schweinle A, Jacobs GA. Assessing posttraumatic stress disorder with or without reference to a single, worst traumatic event: Examining differences in factor structure. Psychological Assessment. 2009; 21:629-634. [PubMed: 19947796]

Goldberg DP, Krueger RF, Andrews G. Emotional disorders: Cluster 4 of the proposed meta-structure for DSM-V and ICD-11. Psychological Medicine. 2009; 39:2043-2059. [PubMed: 19796429]

Herman, J. Trauma and recovery. Basic Books; New York: 1992.

Holzinger KJ, Swineford F. The bi-factor model. Psychometrika. 1937; 2:41-54.

$\mathrm{Hu}$ L, Bentler PM. Cutoff criteria for fit indexes in covariance structure analysis: Conventional criteria versus new alternatives. Structural Equation Modeling. 1999; 6:1-55.

Hurt S, Oltmanns TF. Personality traits and pathology in older and younger incarcerated women. Journal of Clinical Psychology. 2002; 58:457-464. [PubMed: 11920697]

Littlefield AK, Sher KJ, Wood PK. Is "maturing out" of problematic alcohol involvement related to personality change? Journal of Abnormal Psychology. 2009; 118:360-374. [PubMed: 19413410]

Keen SM, Kutter CJ, Niles BL, Krinsley KE. Psychometric properties of PTSD Checklist in sample of male veterans. Journal of Rehabilitation Research and Development. 2008; 45:465-474. [PubMed: 18629754]

Kessler RC, Chiu WT, Demler O, Merikangas KR, Walters EE. Prevalence, severity, and comorbidity of 12-month DSM-IV disorders in the National Comorbidity Survey Replication. Archives of General Psychiatry. 2005; 62:617-627. [PubMed: 15939839]

King DW, King LA, Erickson DJ, Huang MT, Sharkansky EJ, Wolfe J. Posttraumatic stress disorder and retrospectively reported stressor exposure: A longitudinal prediction model. Journal of Abnormal Psychology. 2000; 109:624-33. [PubMed: 11195986]

Kramer MD, Krueger RF, Hicks BM. The role of internalizing and externalizing liability factors in accounting for gender differences in the prevalence of common psychopathological syndromes. Psychological Medicine. 2008; 38:51-61. [PubMed: 17892625] 
Krueger RF. The structure of common mental disorders. Archives of General Psychiatry. 1999; 56:921-926. [PubMed: 10530634]

Krueger RF, Chentsova-Dutton YE, Markon KE, Goldberg D, Ormel J. A cross-cultural study of the structure of comorbidity among common psychopathological syndromes in the general health care setting. Journal of Abnormal Psychology. 2003; 112:437-447. [PubMed: 12943022]

Krueger RF, Skodol AE, Livesley WJ, Shrout PE, Huang Y. Synthesizing dimensional and categorical approaches to personality disorders: Refining the research agenda for DSM-V Axis II. International Journal of Methods in Psychiatric Research. 2007; 16:S65-S73. [PubMed: 17623397]

Krueger RF, South SC. Externalizing disorders: Cluster 5 of the proposed meta-structure for DSM-V and ICD-11. Psychological Medicine. 2009; 39:2061-2070. [PubMed: 19796431]

Kubany ES, Haynes SN, Leisen MB, Owens JA, Kaplan AS, Watson SB, et al. Development and preliminary validation of a brief-broad-spectrum measure of trauma exposure: The Traumatic Events Life Questionnaire. Psychological Assessment. 2000; 12:210-224. [PubMed: 10887767]

Lenzenweger, MF.; Clarkin, JF., editors. Major theories of personality disorder. 2nd ed.. Guilford Press; New York: 2005.

Lenzenweger MF, Lane MC, Loranger AW, Kessler RC. DSM-IV personality disorders in the National Comorbidity Survey Replication. Biological Psychiatry. 2007; 62:553-564. [PubMed: 17217923]

Livesley WJ, Jang KL. Differentiating normal, abnormal, and disordered personality. European Journal of Personality. 2005; 19:257-268.

Livesley WJ, Jang KL, Vernon PA. Phenotypic and genetic structure of traits delineating personality disorder. Archives of General Psychiatry. 1998; 55:941-948. [PubMed: 9783566]

Markon KE. Modeling psychopathology structure: A symptom-level analysis of Axis I and II disorders. Psychological Medicine. 2010; 11:1-16.

Marsh HW, Hau K-T, Wen Z. In search of golden rules: Comment on hypothesis-testing approaches to setting cutoff values for fit indexes and dangers in overgeneralizing Hu and Bentler's (1999) findings. Structural Equation Modeling. 2004; 11:320-341.

Marsh HW, Muthén B, Asparouhov T, Lüdtke O, Robitzsch A, Morin AJS, Trautwein U. Exploratory structural equation modeling, integrating CFA and EFA: Application to students' evaluations of university teaching. Structural Equation Modeling. 2009; 16:439-476.

McCrae RR, Zonderman AB, Costa PT, Bond MH, Paunonen SV. Evaluating replicability of factors in the Revised NEO Personality Inventory: Confirmatory factor analysis versus Procrustes rotation. Journal of Personality and Social Psychology. 1996; 70:552-566.

McGlashan TH, Grilo CM, Skodol AE, Gunderson JG, Shea MT, Morey LC, et al. The collaborative longitudinal personality disorders study: Baseline Axis I/II and II/II diagnostic co-occurrence. Acta Psychiatrica Scandinavia. 2000; 102:256-264.

Miller MW, Fogler JM, Wolf EJ, Kaloupek DG, Keane TM. The internalizing and externalizing structure of psychiatric comorbidity in combat veterans. Journal of Traumatic Stress. 2008; 21:5865. [PubMed: 18302181]

Mulder RT, Joyce PR. Temperament and the structure of personality disorder symptoms. Psychological Medicine. 1997; 27:99-106. [PubMed: 9122314]

Muthén, LK.; Muthén, BO. Mplus user's guide. 5th ed.. Author; Los Angeles, CA: 1998-2009.

Shedler J, Westen D. Refining personality disorder diagnosis: Integrating science and practice. American Journal of Psychiatry. 2004a; 161:1350-1365. [PubMed: 15285958]

Shedler J, Westen D. Dimensions of personality pathology: An alternative to the five-factor model. American Journal of Psychiatry. 2004b; 161:1743-1754. [PubMed: 15465966]

Slade T, Watson D. The structure of common DSM-IV and ICD-10 mental disorders in the Australian general population. Psychological Medicine. 2006; 36:1593-1600. [PubMed: 16882356]

Southwick SM, Yehuda R, Giller EL. Personality disorders in treatment-seeking combat veterans with posttraumatic stress disorder. American Journal of Psychiatry. 1993; 150:1020-1023. [PubMed: 8317570] 
Vollebergh WA, Iedema J, Bijl RV, de Graaf R, Smit F, Ormel J. The structure and stability of common mental disorders: The NEMESIS study. Archives of General Psychiatry. 2001; 58:597603. [PubMed: 11386990]

Watson D. Rethinking the mood and anxiety disorders: A quantitative hierarchical model for DSM-V. Journal of Abnormal Psychology. 2005; 114:522-536. [PubMed: 16351375]

Weathers, FW.; Litz, BT.; Herman, DS.; Huska, JA.; Keane, TM. The PTSD Checklist: Reliability, validity, and diagnostic utility.. Paper presented at the annual meeting of the International Society for Traumatic Stress Studies; San Antonio, TX. Oct. 1993

Widiger TA, Mullins-Sweatt SN. Five-factor model of personality disorder: A proposal for DSM-V. Annual Review of Clinical Psychology. 2008; 5:115-138.

Widiger TA, Simonsen E. Alternative dimensional models of personality disorder: Finding a common ground. Journal of Personality Disorders. 2005; 19:110-130. [PubMed: 15899712]

Wolf EJ, Miller MW, Krueger RF, Lyons MJ, Tsuang MT, Koenen KC. PTSD and the Genetic Structure of Comorbidity. Journal of Abnormal Psychology. 2010; 119:320-330. [PubMed: 20455605]

Yung Y, Thissen D, McLeod LD. On the relationship between the higher-order factor model and the hierarchical factor model. Psychometrika. 1999; 64:113-128. 


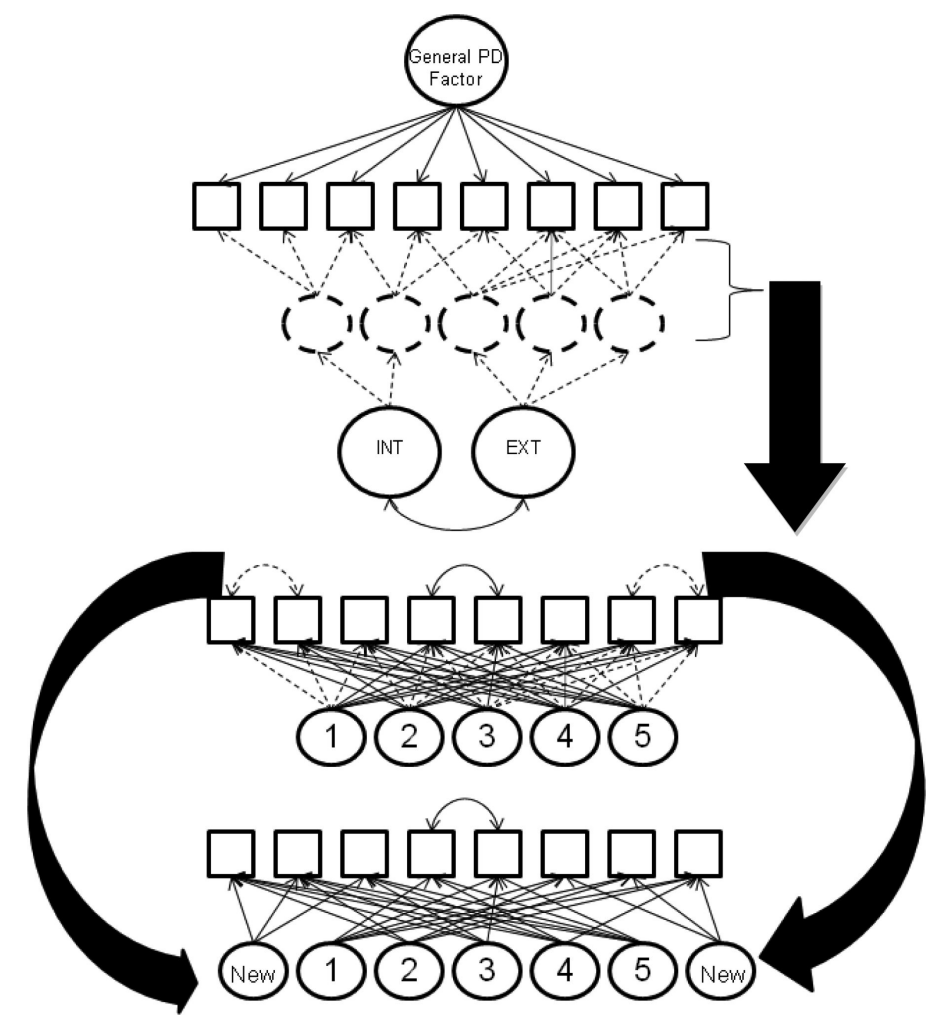

Figure 1.

The panels provide an overview of the process of developing the final bifactor model (shown in Figure 2). Circles denote latent variables and squares reflect observed indictors. Solid lines show aspects of the model that were determined a priori while dotted lines indicate that the nature of a given model component (i.e., the number of trait factors, the loadings) was determined through exploratory methods (shown in the middle panel), even though the general structure of the model was hypothesized a priori. The middle and bottom panels provide a simplified example of how the exploratory structural equation modeling (ESEM) was used (middle panel) to develop the trait portion of the bifactor model (bottom panel). The ESEM revealed several modification indices that suggested residual covariances that were not justifiable as simple method effects (shown by the dotted, curved, double headed arrows in the middle panel) and one modification index that reflected a true method effect. In the bifactor model that was specified subsequently, the residual covariances not due to method effects were specified as new factors in the model and non-significant factor loadings (indicated by dotted loadings in the middle panel) were eliminated, as shown in the bottom panel. 


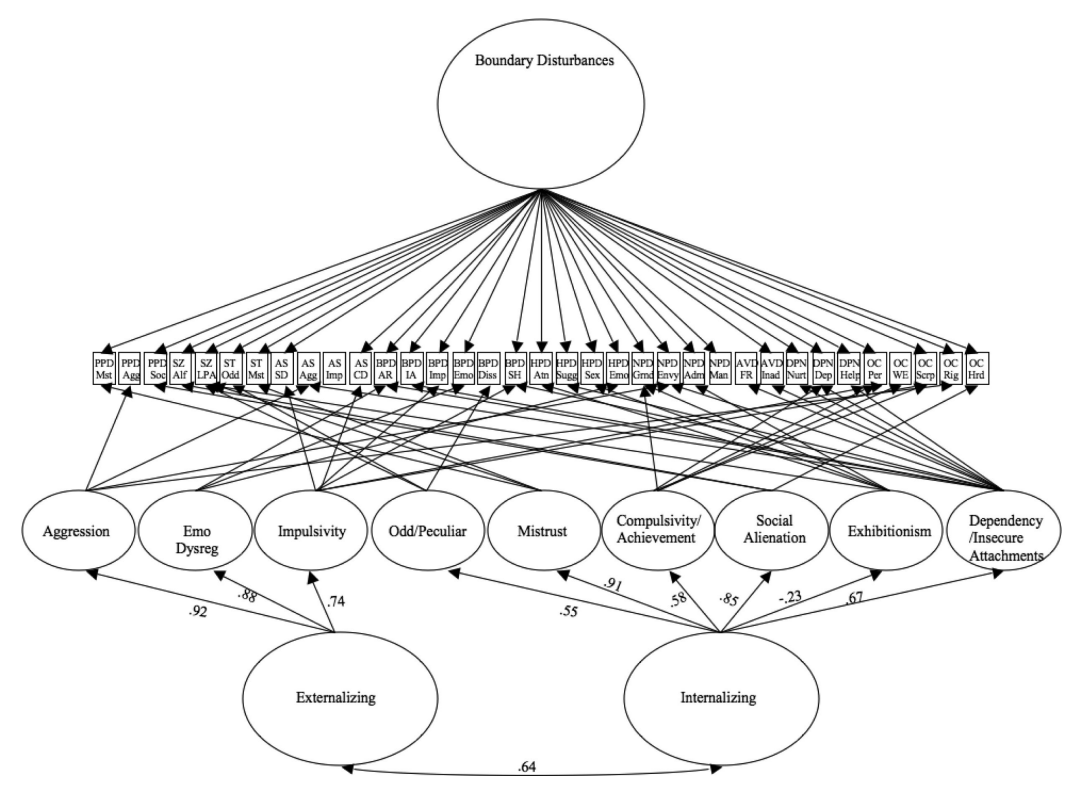

Figure 2.

The Figure shows the final model of the structure of Axis II disorders. Estimates of the structural associations are provided in the measure, factor loadings and indicator error are provided in Table 4. A single correlated residual was estimated in the model (see Table 3), but is not shown here. All abbreviations are provided in Supplemental Table 1. 
Table 1

Results of Exploratory Structural Equation Modeling (ESEM) of SNAP Parcels

\begin{tabular}{lccccc}
\hline Model & Chi Square $(\boldsymbol{d} f)$ & RMSEA & RMR & CFI & TLI \\
\hline 2 Factor & $2012.61^{* * *}{ }^{*}(526)$ & .11 & .10 & .61 & .56 \\
3 Factor & $138.82^{* * *}{ }^{*}(493)$ & .09 & .07 & .77 & .72 \\
4 Factor & $984.26^{* * *}{ }^{*}(461)$ & .07 & .04 & .86 & .82 \\
5 Factor & $864.21^{* * *}{ }_{(430)}$ & .06 & .04 & .89 & .84 \\
6 Factor & $704.36^{* * *}{ }_{(400)}$ & .06 & .03 & .92 & .88 \\
5 Factor with & $800.43^{* * *}(429)$ & .06 & .04 & .90 & .87 \\
1 corr resid. & & & & & \\
\hline
\end{tabular}

Note. SNAP = Schedule for Nonadaptive and Adaptive Personality; corr resid $=$ correlated residual $d f=$ degrees of freedom; RMSEA $=$ root mean square error of approximation; RMR = standardized root mean square residual; CFI = comparative fit index; TLI = Tucker-Lewis index. ESEM was conducted with the maximum likelihood estimator.

$* * * \quad p<.001$ 


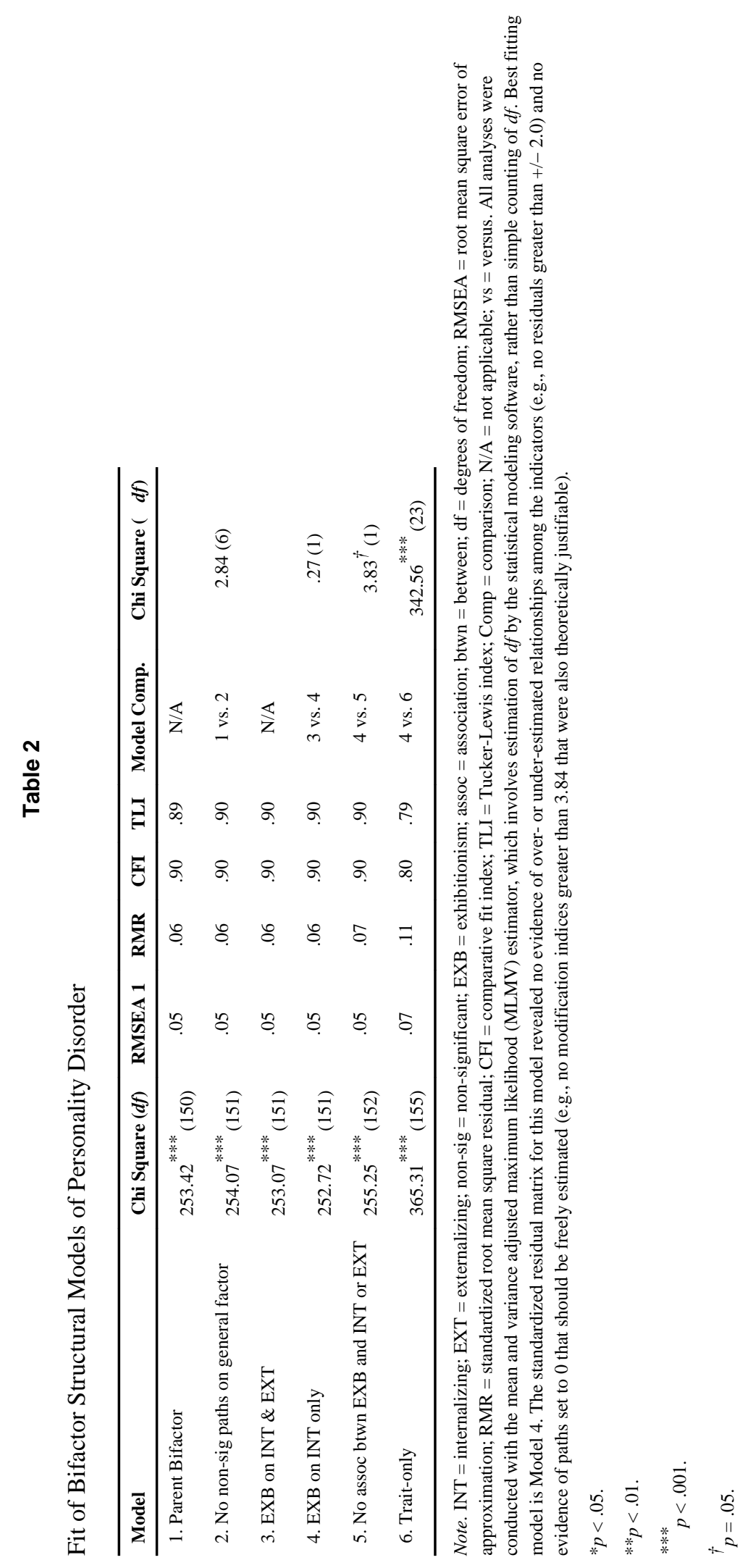

Personal Disord. Author manuscript; available in PMC 2015 January 09. 


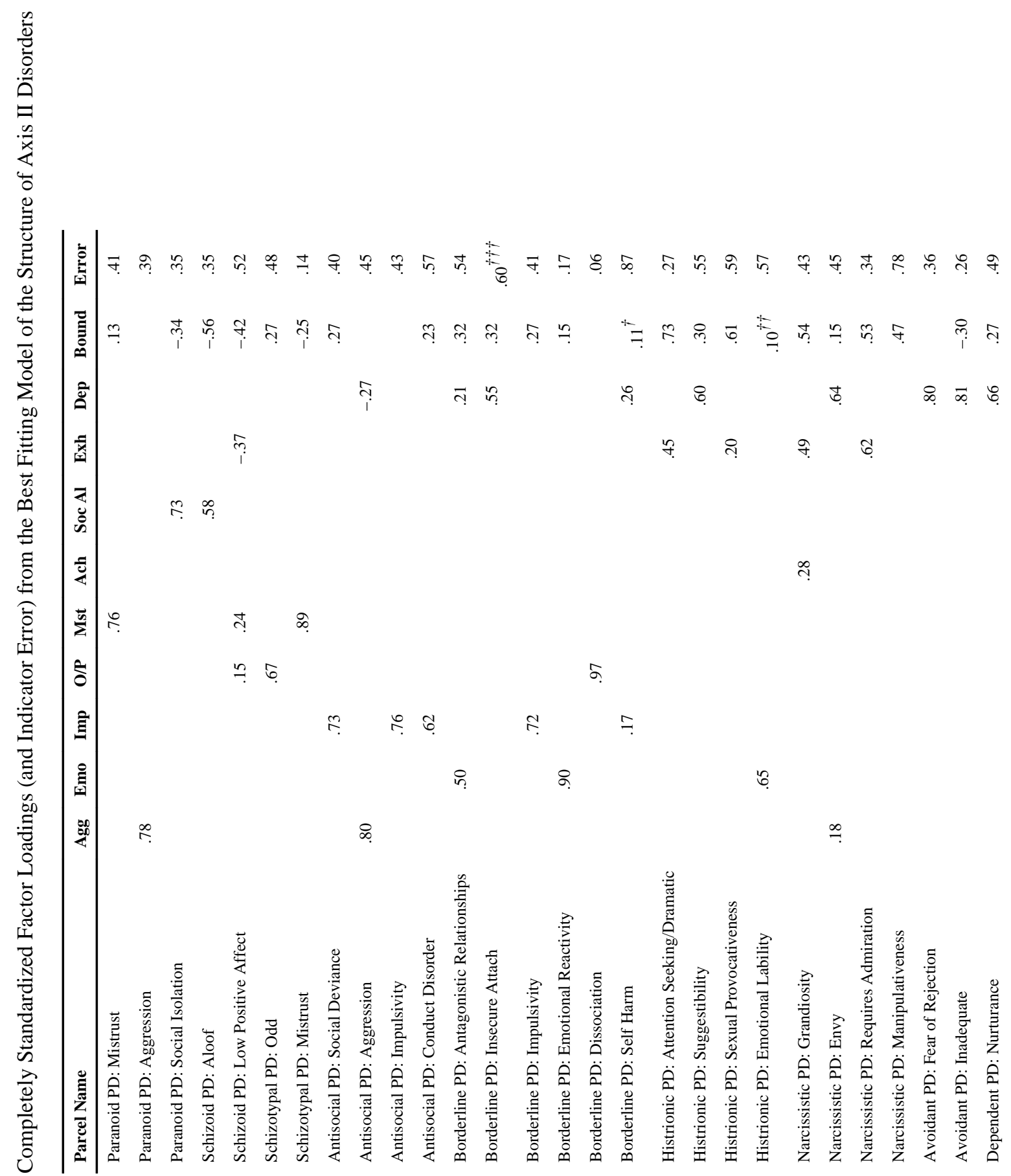



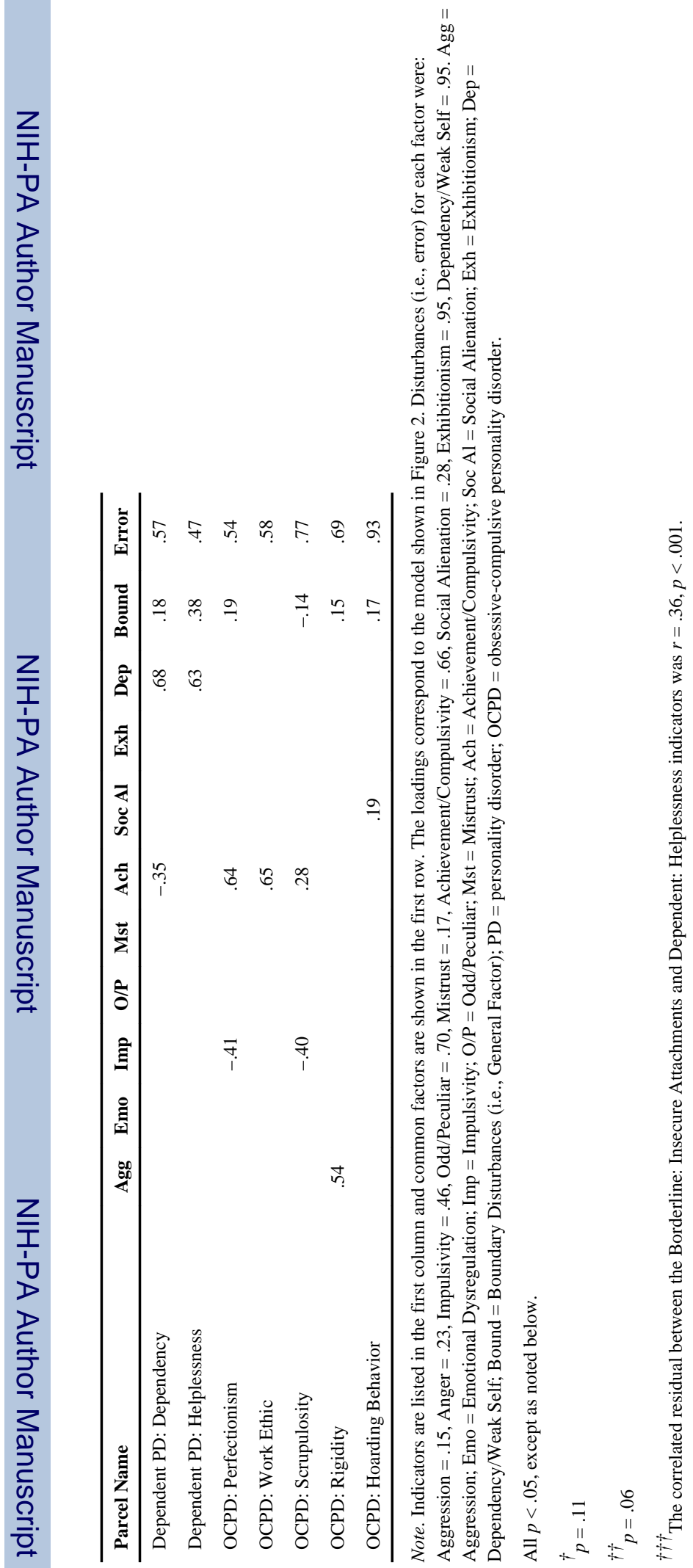

Personal Disord. Author manuscript; available in PMC 2015 January 09. 